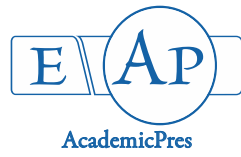

\title{
Generic Salmonella in Asymptomatic Adult Volunteers: Occurrence, Antibiogram, Extended-Spectrum $\beta$-Lactamase Production and Carbapenem Resistance
}

\author{
Chiamaka F. OKPALANWA ${ }^{1}$, Madubuike U. ANYANWU ${ }^{2 *}$, \\ Mumuni A. MOMOH${ }^{1}$, Petra O. NNAMANI ${ }^{1}$, Anthony A. ATTAMA ${ }^{1}$ \\ ${ }^{1}$ University of Nigeria, Faculty of Pharmaceutical Sciences, Pharmaceutics Department, Nsukka, Nigeria; chiamaka.okpalanwa@unn.edu.ng; \\ audu.momoh@unn.edu.ng;petra.nnamani@unn.edu.ng; anthony.attama@unn.edu.ng \\ ${ }^{2}$ Univeristy of Nigeria, Microbiology Unit, Department of Veterinary Pathology and Microbiology, Nsukka, \\ Nigeria; madubuike.anyanwu@unn.edu.ng (*corresponding author)
}

\begin{abstract}
This study was conducted to isolate generic Salmonella from stool of apparently-healthy/asymptomatic adult volunteers in Ankpa North-central Nigeria, and to determine antibacterial susceptibility, extended-spectrum $\beta$-lactamase production and carbapenem resistance. About a gram of stool was collected from 295 randomly-selected apparently-healthy/asymptomatic adult volunteers in Ankpa Kogi State, North-central Nigeria. Isolation of Salmonella was done by pre-enrichment with buffered peptone water, enrichment with Rapapport-Vassiliadis and sub-culturing on Salmonella-Shigella and MacConkey agar. Identification of the isolates to genus level was done using standard biochemical tests. Resistance of the isolates was determined by disc diffusion method. Production of ESBL was assessed by combination disc method while carbapenem resistance was determined using meropenem disc screening method. Nine (3.1\%) out of 295 samples, gave positive growth of Salmonella. Of these 9 isolates, all were carbapenem (meropenem)-resistant and 1 (11.1\%) was ESBL-producer. Among the isolates, all were resistant to ciprofloxacin, ofloxacin, norfloxacin, ampicillin, cefotaxime, ceftazidime, gentamicin and streptomycin, 5 (55.6\%) to sulphamethoxazole-trimethoprim, 7 (77.8\%) to amoxicillin-clavulanic acid and aztreonam, 6 $(66.7 \%)$ to nitrofurantoin and $8(88.9 \%)$ to tetracycline. All the isolates were resistant to $\geq 5$ classes of antibacterial agents. Mean multiple antibacterial indexes of the isolates were 0.68 (range 0.79 - 1.00). Apparently-healthy/asymptomatic adults in Ankpa North-central Nigeria, are potential reservoirs and disseminators of multi- and extensive-drug resistant salmonellae.
\end{abstract}

Keywords: asymptomatic adult; carbapenem resistance; extended-spectrum $\beta$-lactamase production; extensive drug resistance; Salmonella

\section{Introduction}

Salmonella, a member of Enterobacterales is a natural inhabitant of intestinal tract in humans and animals and thus found in various ecological niches such as vegetables, food, water, soil, etc (Khan et al., 2014; Adeolu et al., 2016). Salmonella is facultative-pathogenic especially following immune suppression associated with intercurrent /concurrent infections, malnutrition, water deprivation, prolonged antimicrobial therapy and extremes of age (Lugito and Cucunawangsi, 2017). These conditions facilitate faecal discharge of Salmonella which is uncommon in healthy individuals (Torkan et al., 2015; Im et al., 2016). Antibacterial-resistant (ABR) salmonellae represent a huge threat to global public health (Lugito and Cucunawangsi, 2017). Although most non-typhoidal Salmonella (NTS) infections are self-limiting, ABR non-typhoidal salmonellae, the most frequent cause of foodborne illness in humans and animals are associated with 93.8 million cases of invasive gastroenteritis resulting in up to $35 \%$ hospitalization and $28 \%$ mortality rates per year worldwide while ABR typhoidal salmonellae are associated with 26 million 
384

cases/episodes of enteric fever and approximately 200,000 deaths per year globally (Crump and Heyderman, 2005; Balasubramanian et al., 2018; Britto et al., 2018; Whistler et al., 2018; Klemm et al., 2018). In addition, extra-intestinal focal infections such as bacteraemia, reactive arthritis and osteomyelitis associated with ABR salmonellae, are increasingly being reported (Crump and Heyderman, 2005; Kariuki et al., 2015; Balasubramanian et al., 2018; Whistler et al., 2018). Nowadays, treatment of Salmonellaassociated infections is becoming increasingly difficult due to the global spread of multidrug-resistant (MDR) strains exhibiting resistance to the first-line (chloramphenicol, sulphamethoxazole-trimethoprim [SXT], ampicillin) and second-line (quinolones and $3^{\text {rd }}$-generation cephalosporins [3GCs]) drugs recommended for managing salmonellosis (Le Hello et al., 2013; Kariuki et al., 2015; Whistler et al., 2018). The 3GCs (e.g., ceftazidime, cefotaxime, cefpodoxime) are critically-important drugs of the highest priority in managing serious infections associated with MDR salmonellae (Le Hello et al., 2013; Kariuki et al., 2015; WHO, 2017).

There is increased interest in ABR salmonellae, particularly the 3GC-resistant strains because these organisms exhibit resistance to all classes of antibacterial agents recommended for managing salmonellosis, including aminoglycosides and all $\beta$-lactams except carbapenems (such as meropenem), thereby jeopardizing 3GC therapy in infected individuals/carriers (Le Hello et al., 2013; Kariuki et al., 2015). As a result, these MDR salmonellae are associated with 27 million disability adjusted life years (DALYs) and 30\% of all diarrhoeal DALYs (Moudgil et al., 2017). As in Enterobacterales, resistance to 3GCs in Salmonella is mediated mainly by extended-spectrum $\beta$ lactamases (ESBL) inhibited by clavulanic acid and/or Ampicllinase C (AmpC) which is not inhibited by clavulanic acid (Rawat and Nair, 2010; Klemm et al., 2018; van Duijkeren et al., 2018). ESBL also confers crossresistance to many classes of antibacterial agents such as tetracycline, and all the classes of drugs recommended for treating Salmonella infection (Rawat and Nair, 2010; van Duijkeren et al., 2018). Carbapenems (such as meropenem) are last-resort critically-important drugs of high priority used for treating infections associated with MDR, 3GCresistant and ESBL-producing (ESBL-P) salmonellae (WHO, 2017; Klemm et al., 2018). Carbapenem-resistant salmonellae are extensively drug-resistant (XDR) exhibiting resistance to virtually all available antibacterial agents, and these organisms have the propensity to spread fast within the public due to location of genes encoding carbapenem resistance on mobile genetic elements (Logan and Weinstein, 2017). Thus, the World Health Organization (WHO) recently classified 3GC- and CPN-resistant salmonellae as "critical priority" pathogens which pose threat to health of humans and animals and against which new management strategies and researches documenting their occurrence in different reservoirs is urgently needed (WHO, 2017).

In Nigeria, the use of antibacterial agents, including the critically-important ones like 3GCs and CPNs, in management of human infections (especially diarrhoeic cases with and without fever) is uncontrolled (Akinyemi et al., 2015; Anyanwu et al., 2017; Anibijuwon et al., 2018). Previous treatment with different types of antibacterial agents, including 3GCs and carbapenems, and visitation to hospitals, are factors that facilitate colonization of individuals by ABR salmonellae (Lugito and Cucunawangsi, 2017; Whistler et al., 2018; Adikwu et al., 2018). Animals are also important reservoirs of 3GC- and carbapenemresistant organisms (Crump and Heyderman, 2015; Whistler et al., 2018), and reports showed that these organisms are haboured by livestock in Nigeria (Jajere et al., 2015; Ejikeugwu et al., 2017; Ojo et al., 2018; Anyanwu et al., 2018). Ankpa is a heavily-populated cosmopolitan agrarian town located in Kogi State North-central, Nigeria. There is poor environmental sanitation in Ankpa (defeacation in open air is common, poor waste disposal, etc) and majority of persons resident in this town have poor personal hygiene (Adikwu et al., 2018). Consumption of raw and/or undercooked contaminated meat/plant and/or associated products, drinking of contaminated water as well as direct and/or indirect contact with contaminated animal/plant products, infected individual/carrier and/or contaminated formites, are putative risks for acquisition of ABR salmonellae (Crump and Heyderman, 2015; Adikwu et al., 2018). Thus some residents in Ankpa may habour ABR salmonellae, including ESBL-P, multidrug- and/or carbapenem-resistant strains that could jeopardize antimicrobial therapy. These organisms haboured by asymptomatic/apparently-healthy carriers could be discharged through the stool into the environment (where Salmonella easily survives and proliferates), thus serving as reservoirs and disseminators of genes encoding multiand/or extensive-drug resistance (Abdallah et al., 2017; van Duijkeren et al., 2018). These genes present in the discharged salmonellae could be acquired by other pathogenic human and/or animal bacteria, thereby complicating infection and compromising antibacterial therapy in infected individuals (Torkan et al., 2015; Anyanwu et al., 2018). Assessing the faecal carriage of salmonellae and determining the antibacterial susceptibility of these organisms, is crucial for targeted empiric therapy and for devising strategies for controlling their spread (Crump and Heyderman, 2015; Im et al., 2016).

In the literature, there are several reports on occurrence of ABR salmonellae, including ESBL-P and carbapenemresistant strains, in stool specimens of sick individuals in many parts of the world (Kemal et al., 2015; Ramachandran et al., 2017; Fernandez et al., 2018) as well as in Nigeria (Rotimi et al., 2008; Eze et al., 2016; Abdallah et al., 2017); but studies on faecal carriage of ABR salmonellae in asymptomatic/clinically-healthy human carriers are rather scanty and included the reports in Guinea Bisau/Togo (Im et al., 2016), Ethiopia (Addis et al., 2017), and southwest Nigeria (Smith et al., 2008). Few other reports in South Africa (Richardson and Koornhof, 1965), Togo (Bockemuhl et al., 1976; 1977), Ghana (Feglo et al., 2004) and southwestern Nigeria (Olusanya et al., 1990; Odugbemi and Egri-Kwaji, 1991) focused only on faecal carriage of Salmonella by clinically-healthy/asymptomatic individuals. None of the Nigerian studies assessed ESBL 
production or carbapenem resistance. The potential of clinically-healthy/asymptomatic individuals in Ankpa Kogi State, North-central Nigeria, as reservoirs and disseminators of ABR/ESBL-P/carbapenem-resistant salmonellae has remained uninvestigated. This study was therefore undertaken to determine the occurrence of Salmonella in stool of asymptomatic/clinically-healthy adult residents in Ankpa North-central Nigeria, and to determine the antibacterial susceptibility, ESBL-production and carbapenem resistance.

\section{Materials and Methods}

\section{Study area}

This study was done in Ankpa town, the headquarters of Ankpa Local Government Area, Kogi State North-central Nigeria. Ankpa is geographically located at coordinates at $7^{\circ}$ $22^{\prime} 14^{\prime \prime} \mathrm{N} 7^{\circ} 37^{\prime} 31^{\prime \prime}$. Ankpa has a population of 267,353 (NPC, 2007) with majority of these involved in agricultural activities.

\section{Ethical approval}

Permission to conduct the study was obtained from the Research Ethics Committee of the Pharmaceutics Department, University of Nigeria, Nsukka, and the Medical Research Ethics Committee of the University of Nigeria. The study was conducted in accordance with the code of ethics of the World Medical Association Declaration of Helsinki (WMA, 2013). Written informed consent was obtained from all participants involved in the study and no identifiable data reported.

\section{Sampling}

This cross-sectional study was conducted between January and April, 2018. A total of 295 apparently-healthy adult volunteers ( $\geq 18$ years old) of both sexes who gave written informed consent in accordance with the declaration of Helsinki (WMA, 2013), were randomlyselected. These selected volunteers are residents (who had lived for $\geq 1$ year) of Ankpa and clinicallyhealthy/asymptomatic (without diarrhoea, fever or any other obvious symptom of illness). Each of the selected volunteer was given a clean sterile universal bottle and taught how to collect stool sample aseptically. They were asked to collect about 1 gram of stool sample in the morning and deliver to the researcher within 1 hour to avoid deterioration. Each subject collected and submitted a nonduplicate stool sample of about a gram. Sample was collected from each subject only once. The samples were transported in ice packs to the Microbiology Laboratory, Department of Veterinary Pathology and Microbiology, University of Nigeria, Nsukka and processed within 48 hours of collection.

\section{Isolation and identification of generic salmonellae}

A loopful of the stool samples were inoculated into buffered peptone water (BPW) for pre-enrichment and incubated at $37^{\circ} \mathrm{C}$ for 24 hours in ambient air. Then, a loopful of the BPW broth culture was inoculated into Rapapport-Vassiliadis (RV) medium for enrichment and incubated at $42{ }^{\circ} \mathrm{C}$ for 18 hours in ambient air. A loopful of the RV broth culture was sub-cultured on Salmonella-
Shigella agar (SSA) and MacConkey agar (MCA) and incubated at $37{ }^{\circ} \mathrm{C}$ for 24 hours in ambient air. Two putative Salmonella colonies (tan-coloured/colourless [nonlactose-fermenting] colonies with or without black center on SSA and tan-coloured or colourless colonies on MCA) of each isolate were purified by sub-culturing on SSA and incubated appropriately. Pure cultures of the isolates were inoculated onto nutrient agar slant, incubated at $37^{\circ} \mathrm{C}$ in ambient air and stored in refrigerator at $4{ }^{\circ} \mathrm{C}$ as stock cultures until needed for further analysis. Phenotypic characterization of the isolates to genus level was done by subjecting them to various tests such as oxidase, urease, citrate and triple sugar iron agar tests and fermentation of arabinose, sucrose, maltose, mannitol, mannose, rhamnose, xylose and dulcitol as per standard recommendation (Hendrikssen, 2003).

Determination of antibacterial susceptibility of generic salmonellae from healthy adults

Antibacterial susceptibility profiles of the generic Salmonella isolates was determined by the disc diffusion method on Mueller-Hinton agar following Clinical and Laboratory Standards institute (CLSI) (2018a) guidelines using 13 discs (Oxoid ${ }^{\mathrm{TM}}$ ) impregnated with different antibacterial agents belonging to 7 classes: $\beta$ lactams/cephalosporins - ceftazidime (CTZ; $30 \mu \mathrm{g})$, cefotaxime (CTX; $30 \mu \mathrm{g}$ ), ampicillin (AMP; $10 \mu \mathrm{g}$ ) and aztreonam (AZT; $30 \mu \mathrm{g}), \beta$-lactam combination agents amoxicillin/clavulanic acid (AMC; 20/10 $\mu \mathrm{g}$ ), tetracycline (TET; 30 $\mu$ ), aminoglycosides - streptomycin (STR; $10 \mu \mathrm{g}$ ) and gentamicin (GEN; $10 \mu \mathrm{g})$, fluoroquinolones ciprofloxacin (CIP; $5 \mu \mathrm{g}$ ), ofloxacin (OFL; $5 \mu \mathrm{g}$ ) and norfloxacin (NOR; $10 \mu \mathrm{g}$ ), nitroheterocyclics nitrofurantoin (NIT; $300 \mu \mathrm{g}$ ), and folate inhibitors sulphamethoxazole/trimethoprim (SXT; 25ug). E. coli ATCC (American Type Culture Collection) 25922 was used as reference strain. Results of the antibacterial susceptibility testing were interpreted according to the CLSI (2018b) guidelines for members of the family Enterobacteriaceae. Intermediately-susceptible isolates were classified as resistant. The multiple antibacterial resistance index (MARI) of the isolates was determined using the formula $a / b$ where ' $a$ ' is the number of antibiotics to which an isolate was resistant and ' $b$ ' the number of antibacterial agent the isolate was exposed (Krumperman et al., 1983). Mean MARI was calculated as the ratio of total MARI and total number of resistant isolates. An isolate resistant to antibacterial agents in $\geq 3$ classes/categories was considered MDR (Magiorakos et al., 2012).

\footnotetext{
Detection of extended-spectrum $\beta$-lactamase-producing Salmonella strains

The isolates were screened for ESBL production using the combination disc method (cefpodixime/clavulanic acid $[10: 1 \mu \mathrm{g}]$ and cefpodoxime alone $[10 \mu \mathrm{g}])$ following CLSI (2018a) guidelines. E. coli ATCC 25922 was used as reference strain. An isolate that produced an inhibition zone with a diameter $\geq 5 \mathrm{~mm}$ between the combination disc and cefpodoxime alone was considered ESBL-producer (Anyanwu et al., 2018; CLSI, 2018b).
} 
386

Evaluation of phenotypic carbapenem resistance

Phenotypic carbapenem resistance of the isolates was determined using meropenem (MEM, $10 \mu \mathrm{g}$ ) disc screening following CLSI (2018a) guidelines. E. coli ATCC 25922 was used as reference strain. An isolate that produced an inhibition zone with a diameter $\leq 20 \mathrm{~mm}$ was considered carbapenem-resistant (CLSI, 2018b).

\section{Results}

Occurrence of generic Salmonella in healthy adults

Out of 295 samples cultured, 9 (3.1\%) gave positive growth. All the isolates were rhamnose-positive and xylosepositive.

Antibacterial susceptibility profile of salmonellae from bealthy adults

Out of the 9 Salmonella isolates, all were resistant to
CIP, OFL, NOR, AMP, CAZ, CTX, MEM, GEN and STR, $5(55.6 \%)$ to SXT, 7 (77.8\%) to AMC and AZT, 6 $(66.7 \%)$ to NIT and $8(88.9 \%)$ to TET (Table 1). One (11.1\%) isolate was an ESBL-producer. All the isolates were resistant to $\geq 5$ classes/categories of antibacterial agents (Table 2). Among the isolates, 3 (33.3\%), including the ESBL-producer, were resistant to all the 14 antibacterial agents tested with MARI of $1.00,4(44.4 \%)$ to 12 antibacterial agents with MARI of 0.86 , while 1 (11.1\%) isolate each was resistant to 11 and 13 antibacterial agents with MARI of 0.79 and 0.93 , respectively. Mean MARI for isolates was 0.68 (range $0.79-1.00$ ). The isolates exhibited 7 multiple antibacterial resistance patterns with CIP,CAZ,GEN,AZT,OFX,MEM,NIT,CTX,NOR,AMP, SXT,TET,STR,AMC being the predominant pattern $(n=$ 4).

Table 1. Antibacterial susceptibility profile of 9 generic faecal Salmonella isolates from healthy adults in Ankpa Northcentral Nigeria

\begin{tabular}{|c|c|c|c|}
\hline \multirow{2}{*}{ Antibacterial class } & \multirow{2}{*}{ Antibacterial agent (Concentration) } & \multicolumn{2}{|c|}{ Number (Percentage) of isolates } \\
\hline & & Resistant & Susceptible \\
\hline \multirow{3}{*}{ Fluoroquinolones } & Ciprofloxacin $(5 \mu \mathrm{g})$ & $9(100)$ & $0(0)$ \\
\hline & Ofloxacin $(5 \mu \mathrm{g})$ & $9(100)$ & $0(0)$ \\
\hline & Norfloxacin $(10 \mu \mathrm{g})$ & $9(100)$ & $0(0)$ \\
\hline \multirow{2}{*}{ Aminogylycosides } & Streptomycin $(10 \mu \mathrm{g})$ & $9(100)$ & $0(0)$ \\
\hline & Gentamicin $(10 \mu \mathrm{g})$ & $9(100)$ & $0(0)$ \\
\hline \multirow{5}{*}{$\beta$-lactams } & Ampicillin $(10 \mu \mathrm{g})$ & $9(100)$ & $0(0)$ \\
\hline & Aztreonam $(30 \mu \mathrm{g})$ & $7(77.8)$ & $2(22.2)$ \\
\hline & Ceftazidime $(30 \mu \mathrm{g})$ & $9(100)$ & $0(0)$ \\
\hline & Cefotaxime $(30 \mu \mathrm{g})$ & $9(100)$ & $0(0)$ \\
\hline & Meropenem $(10 \mu \mathrm{g})$ & $9(100)$ & $0(0)$ \\
\hline$\beta$-lactam combination agents & Amoxicillin-clavulanic acid $(25 \mu \mathrm{g})$ & $7(77.8)$ & $2(22.2)$ \\
\hline Tetracycline & Tetracycline $(30 \mu \mathrm{g})$ & $8(88.9)$ & $1(11.1)$ \\
\hline Nitroheterocyclics & Nitrofurantoin $(300 \mu \mathrm{g})$ & $6(66.7)$ & $3(33.3)$ \\
\hline Folate inhibitors & Sulphamethoxazole-trimethoprim $(25 \mu \mathrm{g})$ & $5(55.6)$ & $4(44.4)$ \\
\hline
\end{tabular}

Table 2. Multiple antibacterial resistance profiles, patterns and indices of 9 generic faecal Salmonella isolates from healthy adults in Ankpa Northcentral Nigeria

\begin{tabular}{|c|c|c|c|c|}
\hline $\begin{array}{c}\text { Number of } \\
\text { antibacterial class }\end{array}$ & Resistance pattern (Number of isolates) & $\begin{array}{l}\text { Total number of } \\
\text { isolates (Percentage) }\end{array}$ & $\begin{array}{c}\text { Number of } \\
\text { antibacterial agent }\end{array}$ & $\begin{array}{l}\text { MARI (Total } \\
\text { MARI) }\end{array}$ \\
\hline \multirow{3}{*}{5} & $\begin{array}{c}\text { CIP,CAZ,GEN,AZT,OFX,MEM,NIT,CTX,NOR,AMP, } \\
\text { TET,STR (1) }\end{array}$ & $1(11.1)$ & 12 & $0.86(0.86)$ \\
\hline & $\begin{array}{c}\text { CIP,CAZ,GEN,AZT,OFX,MEM,CTX,NOR,AMP,SXT, } \\
\text { TET,STR (1) }\end{array}$ & $1(11.1)$ & 12 & $0.86(0.86)$ \\
\hline & $\begin{array}{c}\text { CIP,CAZ,GEN,AZT,OFX,MEM,CTX,NOR,AMP,TET, } \\
\text { STR,AMC (1) }\end{array}$ & $1(11.1)$ & 12 & $0.86(0.86)$ \\
\hline \multirow{3}{*}{6} & $\begin{array}{c}\text { CIP,CAZ,GEN,OFX,MEM,CTX,NOR,AMP,SXT,STR, } \\
\text { AMC (1) }\end{array}$ & $1(11.1)$ & 11 & $0.79(0.79)$ \\
\hline & $\begin{array}{c}\text { CIP,CAZ,GEN,AZT,OFX,MEM,NIT,CTX,NOR,AMP, } \\
\text { TET,STR,AMC (1) }\end{array}$ & $1(11.1)$ & 13 & $0.93(0.93)$ \\
\hline & $\begin{array}{c}\text { CIP,CAZ,GEN, } \\
\text { OFX,MEM,NIT,CTX,NOR,AMP,TET,STR,AMC (1) }\end{array}$ & $1(11.1)$ & 12 & $0.86(0.86)$ \\
\hline 7 & $\begin{array}{c}\text { CIP,CAZ,GEN,AZT,OFX,MEM,NIT,CTX,NOR,AMP, } \\
\text { SXT,TET,STR,AMC (3) }\end{array}$ & $3(33.3)$ & 14 & $1.00(3.00)$ \\
\hline
\end{tabular}

CIP: ciprofloxacin; CAZ: ceftazidime; GEN: gentamcicin; OFX: ofloxacin; AZT: aztreonam; MEM: meropenem; NIT: nitrofurantoin; CTX: cefotaxime; NOR: norfloxacin; AMP: ampicillin; TET: tetracycline; AMC: amoxicillin-clavulanic acid; STR: streptomycin; SXT: sulphamethoxazole-trimethoprim; MARI: multiple antibacterial resistance index 


\section{Discussion}

In this study, all the salmonellae were xylose- and rhamnose-negative suggesting they belonged to the NTS species which have been isolated from healthy individuals worldwide (Hendriksen, 2003; Balasubranamian et al., 2018; Fernandez et al., 2018). Although the isolates were not serotyped, they may belong to the invasive NTS serovars such as $S$. typhimurium, $S$. enteritidis etc. associated with intestinal and/or extra-intestinal diseases in humans and animals (Crump and Heyderman, 2015; Balasubranamian et al., 2018; Whistler et al., 2018). The sources of these salmonellae in the sampled individuals may be from contaminated food, meat/plant and/or associated products, contaminated water, and/or or human to human transmission since the hygiene of most Nigerians, including those residing in the study area, could generally be considered poor (Akinyemi et al., 2015; Crump and Heyderman, 2015; Adikwu et al., 2018).

The 3.1\% Salmonella occurrence among 295 volunteers in this study, suggested a low Salmonella occurrence rate in stool specimens of asymptomatic adults in Ankpa Northcentral, Nigeria. This finding is perhaps unsurprising because apparently-healthy individuals were sampled in this study and Salmonella is not frequently discharged in faeces except during antimicrobial therapies and/or in immunosuppressive state (Torkan et al., 2015; Im et al., 2016; Lugito and Cucunawangsi, 2017). The 3.1\% Salmonella occurrence in this study is lower than $13.2 \%$ and $13.63 \%$ occurrence of faecal Salmonella among 53 healthy food-handlers in Lagos Nigeria (Smith et al., 2008), and 22 healthy dairy farm workers in Ethiopia (Addis et al., 2011), respectively. But it is higher than 2, 2.3 and $2.9 \%$ occurrence of faecal Salmonella among 408 clinically-healthy school children in Nigeria (Odugbemi and Egri-Kwaji, 1991), 258 healthy food vendors in Ghana (Feglo et al., 2004) and 245 healthy school children in South Africa (Richardso and Koornhof, 1965). Blockhemul (1976) reported 7\% occurrence of Salmonella in 6051 stool samples of individuals of unspecified health status living in rural regions of Togo. The differences in occurrence of faecal Salmonella in the studies may be related to the method of sampling, isolation/processing, period of sampling, health status and age of sampled individuals, and degree of contamination of individuals (Smith et al., 2008). Whether the sampled individuals were on antibiotic therapy prior to the sampling could also have affected the findings of the studies (Torkan et al., 2015). In this work, the sampled individuals were asked about prior/current infection in order to exclude symptomatic individuals but their history regarding antibacterial therapy (medical history) was not obtained. Therefore, some of these volunteers whose stool yielded Salmonella might be on antibacterial therapy prior to the sampling. It is also possible that some of the subjects had insidious subclinical infection/condition which compromised the immune system thereby resulting in faecal discharge of Salmonella (crump and Heyderman, 2015; Torkan et al., 2015; Im et al., 2016). There is also a possibility that some of the Salmonella-positive stool samples in this study, were obtained from convalescent and chronic carriers/excreters (Crump and Heyderman, 2015; Im et al., 2016).

The 100\% CTX and CAZ resistance in this study indicated complete selection to 3GCs; this finding is probably due to frequent use of these drugs in management of typhoidal and non-typhoidal salmonellosis in Nigeria (Akinyemi et al., 2015). Interestingly, phenotypic ESBL production was detected in only an isolate $(1 / 9 ; 11.1 \%)$ in this study; therefore, the rest of the isolates might have exerted selection to the 3GCs using other non-ESBL mechanisms such as production of AmpC, change in bacterial cell wall permeability, expression of active efflux pumps, etc (van Duijkeren et al., 2018). The production of AmpC by the isolates is the most probable mechanism since $77.8 \%$ of the isolates, including the ESBL-producer, exhibited selection against AMC. Resistance to AMC is mediated by AmpC because of the clavulanic acid component which inhibits ESBL but not AmpC (van Duijkeren et al., 2018). Other mechanisms of AMC such as hyper-production of plasmid-mediated class A $\beta$-lactamases, efflux pumps, etc have also been reported in Enterobacterales (van Duijkeren et al., 2018). There are no studies on faecal carriage of ESBL-producing salmonellae in healthy humans to compare the findings of the hereby study with. However, it is worth to note that Eze et al. (2015) recorded 55.6\% occurrence of ESBL-producers among 36 clinical faecal salmonellae in Anyigba Kogi State, North-central Nigeria using double disc synergy test whereas Akinyemi et al. (2015) used molecular method and reported 16.2\% occurrence of ESBL-producers among 35 clinical faecal Salmonella isolates in Lagos State, Southwest Nigeria. These results are higher than the finding (11.1\%) of this present study. The differences in the occurrence of ESBL-producers in the studies may be due to the differences in the ESBL detection method used, and usage of ESCs in management of diseases in the sampled individuals. In the hereby experiment, the combination disc method employing cefpodoxime and cefpodoxime/clavulanic acid was used; this recommended method is reliable in detecting ESBL production but not as accurate as the molecular method (Anyanwu et al., 2018). Further, over-expression of nonESBL (such as TEM-1 and OXA-1) and loss of porins could result in false ESBL phenotypes in Enterobacterales (Beceiro et al., 2011); therefore, the occurrence of ESBLproducing Salmonella strains may be more or less than as observed in this study.

The finding of complete 3GC selection in this study calls for serious concern because 3GCs are recommended second-line drugs for managing infections associated with salmonellae resistant to the first-line drugs, and moreover 3GC-resistant salmonellae are MDR (as also observed in this study) exhibiting resistance to all $\beta$-lactams (except carbapenems) and to the other classes of antibacterial agents (sulphonamides, trimethoprim, aminoglycosides, fluoroquinolones, tetracycline and chloramphenicol) recommended for managing clinical salmonellosis (Lugito and Cucunawangsi, 2017; Balasubramanian et al., 2018; Klemm et al., 2018). Unfortunately, all the isolates in this study were carbapenem-resistant and they exhibited extensive drug resistance being insusceptible to $\geq 5$ classes of antibacterial agents, including those recommended for 
388

treating Salmonella infections (Magiorakos et al., 2012; Lugito and Cucunawangsi, 2017; Logan and Weinstein, 2017). Thus they are "superbugs" capable of causing intractable infections (Logan and Weinstein, 2017). This finding of complete carbapenem resistance in this study, calls for urgent attention because of possible danger of limited therapy in the sampled individuals since MEM is the last-resort drug for treatment of MDR infections, including ESC-resistant and ESBL-P Salmonella-associated infections (Logan and Weinstein, 2017; Klemm et al., 2018). The MEM resistance in this study suggested production of carbapenemases (not assessed in this study) but there is also the possibility that non-carbapenemase mechanisms such as altered outer membrane proteins, high level production of AmpC and/or ESBL (in the ESBL-producing isolate) and/or reduced permeability of altered porins complemented by hydrolytic action of AmpC and/or ESBL (in the ESBL isolate) were involved in the carbapenem resistance (Logan and Weinstein, 2017; van Duijkeren et al., 2018). There is no report on carbapenem resistance among Salmonella isolates from clinically-healthy individuals to compare the results of this study with. However, the complete carbapenem resistance in this study could be responsible for a higher resistance against all the drugs than that reported among Salmonella isolates from healthy individuals in Ethiopia (Addis et al., 2011) and Lagos Nigeria (Smith et al., 2008). This may also reflect the differences in antibacterial usage in the study areas. The criteria used for classifying isolates as 'resistant' or 'susceptible' may also underline the variations in resistances in the studies. In this experiment, intermediately-susceptible isolates were classified 'resistant' and with regards to aminoglycosides (GEN and STR), susceptible isolates were classified 'resistant' in accordance with the current CLSI (2018) recommendations. The MARI (mean 0.68, range $0.79-1.00)$ in this study suggested that the isolates were frequently exposed to antibacterial agents since MARI values $>0.2$ indicate high contamination risk source where antibacterial agents are often used (Krumperman, 1983).

\section{Conclusions}

This study has shown that a considerable percentage (3.1\%) of clinically-healthy/asymptomatic adults in Ankpa North-central Nigeria, discharge carbapenem-resistant salmonellae in their stool with some of these strains being ESBL-producers. These individuals are potential reservoirs and disseminators of multi- and extensively drug-resistant salmonellae and genes encoding resistance to virtually all antibacterial agents recommended for managing salmonellosis. The presence of multi- and extensively-drug resistant salmonellae in asymptomatic persons in Ankpa, has huge impact on the ecology and epidemiology of antibacterial resistance in Nigeria. Thus, there is need for precautionary use of 3GCs and carbapenems in the management of Salmonella infections in Nigeria. However, further studies to determine the serotypes of the salmonellae and resistance genes haboured by these isolates are recommended.

\section{Conflict of Interest}

The authors declare that there are no conflicts of interest related to this article.

\section{References}

Abdallah HM, Alnaiemi N, Reuland EA, Wintermans BB, Koek A, Abdelwahab AM, ... Vandenbroucke-Grauls CMJE (2017). Fecal carriage of extended-spectrum $\beta$-lactamase- and carbapenemaseproducing Enterobacteriaceae in Egyptian patients with communityonset gastrointestinal complaints: a hospital-based cross-sectional study. Antimicrobial Resistance Infection Control 6:62.

Addis Z, Kebede N, Worku Z, Gezahegn H, Yirsaw A, Kassa T (2011). Prevalence and antimicrobial resistance of Salmonella isolated from lactating cows and in contact humans in dairy farms of Addis Ababa: a cross sectional study. BMC Infectious Diseases 11(1):222.

Adeolu M, Alnajar S, Naushad S, Gupta R (2016). Genome-based phylogeny and taxonomy of the 'Enterobacteriales': proposal for Enterobacterales ord. nov. divided into the families Enterobacteriaceae, Erwiniaceae fam. nov, Pectobacteriaceae fam. nov., Yersiniaceae fam. nov., Hafniaceae fam. nov., Morganellaceae fam. nov., and Budviciaceae fam. nov. International Journal of Systemic and Evolutionary Microbiology 66(12):5575-5599.

Adikwu P, Umeh EU, Iheukwumere CC, Ogbonna IO, Awodi PS, Obande GA (2018).Variation in Salmonellatyphi infection amonglocal populations in Southern Benue, Nigeria. International Journal of Enteric Pathogens 6(4):89-94.

Akinyemi KO, Iwalokun BA, Alafe OO, Mudashiru SA, Fakorede C (2015). bla Salmonella typhi from hospitalized patients in Lagos Nigeria. Infection and Drug Resistance 8:99-106.

Anyanwu MU, Ugwu IC, Onah CU (2018). Occurrence and antibiogram of generic extended-spectrum cephalosporin-resistant and extendedspectrum $\beta$-lactamase-producing enterobacteria in horses. Macedonian Veterinary Reviews 41(2):123-132.

Anibijuwon II, Gbala ID, Adebisi OO (2018). Carbapenem-resistant Enterobacteriaceae among in-patients of tertiary hospitals in Southwest Nigeria. NotulaeScientia Biologicae 10(3):310-317.

Balasubramanian R, Im J, Lee JS, Jeon HJ, Mogeni OD, Kim JH,... Marks F (2018). The global burden and epidemiology of invasive non-typhoidal Salmonella infections. Human Vaccines and Immunotherapeutics 15(6):1421-1426.

Beceiro A, Maharjan S, Gaulton T, Doumith M, Soares NC, Dhanji H,... Woodford N (2011). False extended-spectrum \{beta\}-lactamase phenotype in clinical isolates of Escherichia coli associated with increased expression of OXA-1 or TEM-1 penicillinases and loss of porins. Journal of Antimicrobial Chemotherapy 66(9):2006-2010.

Bockemuhl J (1976). Salmonellosis and shigellosis in Togo (West Africa), 1971-1973. I. Carrier rates in the rural population. Tropenmedizin und Parasitology 27(1):112-120.

Bockemuhl J (1977).Salmonellosis and shigellosis in Togo (West Africa), 1971-1973. Infections in the urban population of Lome.. Tropenmedizin und Parasitology 28(3):377-383. 
Britto CD, Wong VK, Dougan G, Pollard AJ (2018). A systematic review of antimicrobial resistance in Salmonella enterica serovar Typhi, the etiological agent of typhoid. PLoS Neglected Tropical Diseases 12(10):e0006779.

Clinical and Laboratory Standards Institute (CLSI) (2018a). Methods for dilution antimicrobial susceptibility tests for bacteria that grow aerobically. 11th ed. CLSI Standard M07. Wayne, PA: Clinical and LaboratoryStandards Institute.

Clinical and Laboratory Standards Institute (CLSI) (2018b). Performance standard for antimicrobial susceptibility testing 28th ed. CLSI Standard M100, Wayne, PA: Clinical and LaboratoryStandards Institute.

Crump JA, Heyderman RS (2015). A perspective on invasive Salmonella disease in Africa. Clinical Infectious Diseases 61(4):S235S240.

Eze EA, Ocean EO, Eze EN (2015). Prevalence of beta-lactamases in Salmonella and Shigella in different hospitals in Anyigba Kogi State, Nigeria. African Journal of Microbiology Research 9(3):180-184.

Ejikeugwu C, Iroha I, Oguejiofor B, Orji Okoro L, Eluu S, Okafor C , ... Ezeador C (2017). Multidrug resistant metallo- $\beta$-lactamases (MBLs) positive Klebsiella species from cloacal swabs of poultry birds. Journal of Bacteriology and Parasitology 8:305.

Feglo PK, FrimpongEH,Essel-Ahun M (2004). Salmonellae carrier status of food vendors in Kumasi, Ghana. East African Medical Journal 81(7):358-361.

Fernández J, Guerra B, Rodicio MR (2018). Resistance to carbapenems in non-typhoidal Salmonella enterica serovars from humans, animals and food. Veterinary Science 5(2):40.

Hendriksen R (2003). A global Salmonella surveillance and laboratory support project of the World Health Organization: Laboratory Protocols (Isolation of Salmonella), 4th Edition pp 253-278.

Jajere SM, Adamu NB, Atsanda NN, Onyilokwu SA, Gashua MM Hambali IU, Mustapha FB (2015). Prevalence and antimicrobial resistance profiles of Salmonella isolates in apparently healthy slaughtered food animals at Maiduguri central abattoir Nigeria. Asian Pacific Journal of Tropical Diseases 5(12):996-1000.

Im J, Nichols C, Bjerregaard-Andersen M,Sow AG, LøfbergS, Tall A, ... von Kalckreuth V (2016). Prevalence of Salmonella excretion in stool: a community survey in 2 sites, Guinea-Bissau and Senegal. Clinical Infectious Diseases 62(1):S50-S55.

Kariuki S, Gordon MA, Feasey N,Parry CM (2015). Antimicrobial resistance and management of invasive Salmonella disease. Vaccine 33(03):C21-C29.

Kemal J, Sibhat B, Menkir S, Terefe Y, Muktar Y (2015). Antimicrobial resistance patterns of Salmonella in Ethiopia: a review. African Journal of Microbiology Research 9(4):2249-2256.

Khan CMA (2014). The dynamic interactions between Salmonella and the microbiota, within the challenging giche of the gastrointestinal tract. International Scholarly Research Notes http: //dx.doi.org /10.1155/2014/846049.

Klemm EJ, Shakoor S, Page AJ, Qamar FN, Judge K, Saeed DK,...Hasan R (2018). Emergence of an extensively drugresistant Salmonella enterica serovar Typhi clone harboring a promiscuous plasmid encoding resistance to fluoroquinolones and third-generation cephalosporins.
mBio 9(1):e00105-18.

Krumperman PH (1983). Multiple antibiotic resistance indexing of Escherichia coli to identify high-risk sources of fecal contamination of foods. AppliedEnvironmental Microbiology 46(1):165-170.

Le Hello S, Bekhit A, Granier SA, Barua H, Beutlich J,Zając M,... Weill FX (2013). The global establishment of a highly-fluoroquinolone resistant Salmonella enterica serotype Kentucky ST198 strain. Frontiers in Microbiology 18(4):395.

Magiorakos AP, Srinivasan A, Carey RB, Carmeli Y, Falagas ME, Giske CG,... Monnet DL (2012). Multidrug-resistant, extensively drugresistant and pandrug-resistant bacteria: an international expert proposal for interim standard definitions for acquired resistance. Clinical Microbiology and Infection 18(3):268-281.

Moudgil P, Bedi JS, Moudgil AD, Gill JPS, Aulakh RS (2017). Emerging issue of antibiotic resistance from food producing animals in India: Perspective and legal framework. Food Reviews International 34(5):447-462.

Nigerian Population Commission (NPC) (2007). Federal Republic of Nigeria (NPC/FRN). Special FRN, Gazette no 23 on the 2006 population census.

Ojo OE, Schwarz S, Michael GB (2016). Detection and characterization of extended-spectrum $\beta$-lactamase-producing Escherichia coli from chicken production chains in Nigeria. Veterinary Microbiology 15(194):62-68.

Olusanya O, Shonukan OO, Ogwo VA (1990). Childhood carriers of Salmonella and Shigella species in the rural area of Ile-Ife, Nigeria. Ethiopian Medical Journal 28(2):49-52.

Odugbemi T, Egri-Kwaji MT (1991).Screening of children for enteric bacterial pathogens in the outborn neonatal ward in Lagos, Nigeria. European Journal of Epidemiology 7(4):427-430.

Logan LK, Weinstein RA (2017). Epidemiology of carbapenem-resistant Enterobacteriaceae: The impact and evolution of a global menace. Journal of Infectious Diseases 215(S1):S28-36.

Lugito NPH, Cucunawangsi (2017). Antimicrobial resistance of Salmonella enterica serovars Typhi and Paratyphi isolates from a general hospital in Karawaci, Tangerang, Indonesia: A five-year review. International Journal of Microbiology https://doi.org/10.1155/2017/6215136.

Ramachandran A, Shanthi M, Sekar U (2017). Detection of blactX:M extended spectrum beta-lactamase producing Salmonella enterica serotype Typhi in a tertiary care centre. Journal of Clinical Diagnostic Research 11(9):DC21-DC24.

RawatD, NairD (2010). Extended-spectrum $\beta$-lactamases in Gram negative bacteria. Journal of Global Infectious Diseases 2(3):263-274.

Richardson NJ, KoornhofHJ (1965). Salmonellae and Shigellae in a group of Bantu school children in the eastern Transvaal Lowveld. South African Medical Journal 39(5):367-370.

Rotimi VO, Jamal W, Pal T, Sovenned A, Albert MJ (2008). Emergence of CTX-M-15 type extended-spectrum b-lactamase-producing Salmonella spp. in Kuwait and the United Arab Emirates. Journal of Medical Microbiology 57(7):881-886.

Smith SI, Alao F, Goodluck HT, Fowora M, Bamidele M, Omonigbehin E, Coker AO (2008). Prevalence of Salmonella typhi among food handlers from bukkas in Nigeria. British Journal of Biomedical Science 65(3):158-160. 
390

Torkan S, Khamesipour F, Anyanwu MU (2015). Detection of virulence and antibacterial resistance genes in Salmonella isolates from diarrhoeic dogsin Iran. Revue de Medicine Veterinaire 166(7-8):221-228.

van Duijkeren E, Schink AK, Roberts MC, Wang Y, Schwarz S (2018). Mechanisms of bacterial resistance to antimicrobial agents. MicrobiologySpectrum 6(1).

Whistler T, Sapchookul P, McCormick DW, Sangwichian O, Jorakate P, Makprasert S, ... Gregory CJ (2018). Epidemiology and antimicrobial resistance of invasive non-typhoidal salmonellosis in rural Thailand from 2006-2014. PLoSNeglected Tropical Diseases 12(8):e0006718.

World Medical Association (WMA) (2013). World Medical Association Declaration of Helsinki: Ethical principles for medical research involving human subjects. Journal of American Medical Association 310(20):2191-2194.
World Health Organization (WHO). Global priority list of antibioticresistant bacteria to guide research, discovery, and development of new antibiotics http://www.who.int/medicines/publications/WHO-PPLShort_Summary_25FebET_NM_WHO.pdf.2017.

World Health Organization (WHO). Critically important antimicrobials for human medicine. $5^{\text {th }}$ edition. http:// apps.who.int/iris/bitstream/handle/10665/255027/9789241512220e ngpdf;jessionid=E6465D3C02939652305588FF323F0210?sequen $c e=1$. 